\title{
Concurrent use of alcohol interactive medications and alcohol in older adults: a systematic review of prevalence and associated adverse outcomes
}

\author{
Alice E. Holton ${ }^{1 *}$, Paul Gallagher ${ }^{1}$, Tom Fahey ${ }^{2}$ and Gráinne Cousins ${ }^{1}$
}

\begin{abstract}
Background: Older adults are susceptible to adverse effects from the concurrent use of medications and alcohol. The aim of this study was to systematically review the prevalence of concurrent use of alcohol and alcohol-interactive (Al) medicines in older adults and associated adverse outcomes.

Methods: A systematic search was performed using MEDLINE (PubMed), Embase, Scopus and Web of Science (January 1990 to June 2016), and hand searching references of retrieved articles. Observational studies reporting on the concurrent use of alcohol and Al medicines in the same or overlapping recall periods in older adults were included. Two independent reviewers verified that studies met the inclusion criteria, critically appraised included studies and extracted relevant data. A narrative synthesis is provided.

Results: Twenty studies, all cross-sectional, were included. Nine studies classified a wide range of medicines as Al using different medication compendia, thus resulting in heterogeneity across studies. Three studies investigated any medication use and eight focused on psychotropic medications. Based on the quality assessment of included studies, the most reliable estimate of concurrent use in older adults ranges between 21 and 35\%. The most reliable estimate of concurrent use of psychotropic medications and alcohol ranges between 7.4 and $7.75 \%$. No study examined longitudinal associations with adverse outcomes. Three cross-sectional studies reported on falls with mixed findings, while one study reported on the association between moderate alcohol consumption and adverse drug reactions at hospital admission.

Conclusions: While there appears to be a high propensity for alcohol-medication interactions in older adults, there is a lack of consensus regarding what constitutes an Al medication. An explicit list of Al medications needs to be derived and validated prospectively to quantify the magnitude of risk posed by the concurrent use of alcohol for adverse outcomes in older adults. This will allow for risk stratification of older adults at the point of prescribing, and prioritise alcohol screening and brief alcohol interventions in high-risk groups.
\end{abstract}

Keywords: Alcohol, Drug interaction, Alcohol interactive, Older adult, Psychotropic medicines, Adverse outcomes

\footnotetext{
* Correspondence: aliceholton@rcsi.ie

${ }^{1}$ School of Pharmacy, Royal College of Surgeons in Ireland (RCSI), Dublin 2,

Ireland

Full list of author information is available at the end of the article
}

(c) The Author(s). 2017 Open Access This article is distributed under the terms of the Creative Commons Attribution 4.0 International License (http://creativecommons.org/licenses/by/4.0/, which permits unrestricted use, distribution, and reproduction in any medium, provided you give appropriate credit to the original author(s) and the source, provide a link to the Creative Commons license, and indicate if changes were made. The Creative Commons Public Domain Dedication waiver (http://creativecommons.org/publicdomain/zero/1.0/) applies to the data made available in this article, unless otherwise stated. 


\section{Background}

By 2050 , older adults aged $\geq 60$ years are expected to account for $34 \%$ of the population in Europe [1]. While alcohol consumption changes over the life-course, with a decline in consumption in older age, recent evidence from nine UK based prospective cohort studies, have shown that drinking occasions tend to become more frequent among older adults [2]. There is also evidence of a cohort effect, with successive birth cohorts reporting an increase in alcohol consumption across all age-groups, including among older adults [3].

Even at relatively low levels of alcohol consumption, older adults can be vulnerable to harm, with physiological changes exacerbating these harms $[4,5]$. Furthermore, older adults experience a disproportionate burden of alcohol related-harm; in England between 2009 and 2010 , adults aged $\geq 65$ years accounted for approximately $44 \%(461,400)$ of alcohol-related hospital admissions yet comprised of only $17 \%$ of the population $[6,7]$. Alcoholrelated deaths were also highest among those aged 55 to 74 years [4].

Furthermore, the use of multiple medicines is increasing in older adults [8-11]. A recent Irish study reported an increase in polypharmacy from 17.8 to $60.4 \%$ between the years 1997-2012 in older adults aged $\geq 65$ years [11]. Certain medications have the potential to interact with alcohol; these medications are referred to as alcohol interactive (AI) medications [12]. They may interact with alcohol by altering the metabolism (pharmacokinetic) or effects (pharmacodynamic) of alcohol and/or the medication [12]. Certain interactions may occur with any alcohol consumption, whereas other interactions may follow a dose response, with the risk or severity of an interaction increasing with increasing levels of alcohol [13]. AI medications, when combined with alcohol, increase the risk of medical complications such as hypoglycaemia, hypotension, sedation, gastrointestinal bleeds and liver damage, in older adults $[5,12]$. For example, older adults are vulnerable to the sedating effects of alcohol and when combined with central nervous system (CNS) agents, such as psychotropic medications, older adults have an increased risk of sedation and drowsiness [5]. Psychotropic medicines include antidepressants, sedatives/hypnotics, stimulants and neuroleptics, all of which act on the CNS and are commonly prescribed to older adults [14]. Similarly, concurrent use of alcohol with cardiovascular agents, such as vasodilatory agents, increases the risk of hypotension in older adults [5], with concurrent use with non-steroidal antiinflammatory drugs (NSAIDs) increasing the risk of gastrointestinal bleeds [12].

While a recent systematic review has assessed the prevalence of concurrent alcohol use and prescription sedative-hypnotic medicines in middle-aged and older adults, [15] there have been no systematic reviews on the prevalence of concurrent use of alcohol and alcohol interactive medications beyond psychotropic medications and associated adverse outcomes in older adults. An older review did investigate the potential risk of combining alcohol with medications in older adults however the focus of the review was on the pharmacology and mechanism of action involved in alcoholmedication interactions and potential clinical implications of these interactions [5]. Therefore this study aims to systematically review the prevalence of concurrent use of alcohol and alcohol interactive medications in older adults and associated adverse outcomes.

\section{Methods}

This systematic review was performed according to Preferred Reporting Items for Systematic reviews and MetaAnalyses (PRISMA) guidelines [16].

\section{Search strategy}

A comprehensive systematic search was performed using MEDLINE (PubMed), Embase, Scopus and Web of Science. A combination of the following keywords and $\mathrm{MeSH}$ terms were used: "ethanol", "alcohol", "drug interactions", "drug alcohol interaction" and "aged". This search was supplemented by a search in Google Scholar and by hand searching references of retrieved articles. The search was restricted to English language articles and articles published since January 1990 to June 2016.

\section{Study selection and data extraction}

Studies were included if they met the following eligibility criteria: Observational studies reporting on the concurrent use of alcohol and alcohol interactive (AI) medicines in the same or overlapping recall periods in older adults. Studies also had to report on the quantity or frequency of alcohol consumption. We excluded studies which exclusively sampled patients with specific illnesses, or those seeking treatment for alcohol use disorders (AUD) or illicit drug use.

Title and abstracts of identified studies were reviewed by one reviewer $(\mathrm{AH})$ to determine potential eligibility. Full text articles were then reviewed by two reviewers (AH/GC) for those studies considered eligible from title/ abstract, or when it was unclear whether a study met the inclusion criteria. The following data were extracted by two reviewers $(\mathrm{AH} / \mathrm{GC})$ : year of publication, country, study sample, study design, measurement and definition of alcohol interactive (AI) medications, measurement of alcohol consumption, prevalence of alcohol use and AI medication use and prevalence of concurrent alcohol and AI medication use. Adverse outcomes associated with concurrent use of alcohol and AI medications were also extracted if reported. Any uncertainty in relation to 
study eligibility and data extraction was resolved through discussion between two reviewers (GC/AH).

\section{Critical appraisal}

The risk of bias was evaluated, by two reviewers (AH/ GC), using an adapted form of the Newcastle Ottawa cohort scale (NOS) [17]. This amended NOS scale allowed for the evaluation of cross-sectional studies, focussing on the risk of selection bias and information bias, specifically misclassification bias of both exposures (alcohol and AI medications) and the outcome (concurrent use of alcohol and AI medications) across included studies.

\section{Results}

Of the 546 citations identified from this search strategy, 108 full text articles were assessed for eligibility, with 20 studies meeting the inclusion criteria [14, 18-36] (Fig. 1).

\section{Study characteristics}

Ten of the included studies were conducted in Europe $[14,19,21-23,25,27-29,36]$, eight in North America $[18,20,24,30-32,34,35]$ and two in Australia [26, 33]. (Table 1) All studies were cross-sectional [14, 18-36]. Study settings varied across studies with; communitydwelling [14, 19, 21-27, 31, 34-36], both community dwelling or living independently in care facilities [32], general populations $[20,28,33]$, hospital setting [29], retirement communities [18] and participants signed up to a pharmaceutical assistance contract for the elderly [30] reported.

Sample sizes varied from 311 to 83,321 participants $[18,30]$. Men and women were included in all studies, with the exception of one Australian study which only included men [26]. Nine studies reported on a wide range of prescription and/or over the counter (OTC) medicines with potential to interact with alcohol [18-21, 24, 27, 29-31] . Three studies investigated any medication use during the recall period [23, 33, 35], and a further eight focused on psychotropic medications [14, 22, 25, 26, 28, 32, 34, 36]. Of the eight studies focusing on psychotropic medications, five investigated psychotropic medications alone [22, 25, 26, 34, $36]$ and three studies also included analgesics [14, 28, 32].

Of the nine studies focusing on a wide range of AI medications, all studies classified central nervous system (CNS) agents as AI medications (Table 1). Consistent with those studies investigating psychotropic medications $[14,22,25,26,28,32,34,36]$, the following drug classes were classified as AI medications, sedatives/ hypnotics $[18-21,24,27,30,31]$, antidepressants [18-21, 27, 30, 31], opioids/narcotics [18-21, 27, 30, $31]$, anticonvulsants [19-21, 24, 27, 30, 31] and antipsychotics [19-21, 27, 29, 30]. After CNS agents, cardiovascular medicines (CVS) were the most common AI medicines [18-21, 24, 27, 29-31], followed by antidiabetic drugs $[18,20,21,24,27,29-31]$, warfarin [18-21, 24, 27, 30, 31], gastrointestinal agents [18-21, $24,30,31]$, non-steroidal anti-inflammatory drugs (NSAIDs) [18-21, 29-31], antibiotics/anti-infectives [21, 24, 27, 29-31] and anti-histamines [19-21, 30].

\section{Quality assessment}

The methodological quality of the included studies is detailed in Table 2. The external validity was high in 4 studies, as they reported on random population samples of community dwelling older adults aged $\geq 60$ or $\geq 65$ years $[21,24,27]$ or in the case of Breslow et al., a random population sample with oversampling of older adults aged $\geq 60$ years [20]. Ten studies were considered to have moderate external validity; five studies sampled random community dwelling older adults but with age restrictions which may have introduced selection bias $[14,19,25,26,31]$ such as including adults aged between 53 and 75 years [25] or only including adults $\geq 75$ years [19]. A further five studies reported on random population samples of community dwelling adults with subgroup analysis of older adults, however they did not report oversampling of older adults [22, 23, 28, 33, 34]. The six remaining studies were considered to have poor external validity, as the risk of selection bias is considered to be high $[18,29,30,32,35,36]$. For example, Pringle et al. recruited enrollees in the Pennsylvania pharmaceutical assistance contract for the elderly, which may not be representative of all older adults as the members are older (mean age 78.8 years) and more likely to be female and white with multiple chronic conditions [30].

Internal validity was assessed by evaluating the potential risk of misclassification bias for both exposure and outcome across studies. Ten studies were considered to have a low risk of misclassifying exposure to medications which have the potential to interact with alcohol, as they used prescription claims data or in house inventories where they recorded details from the labels of medication containers or prescriptions and provided references supporting the inclusion of medicines as potentially alcohol interactive (Table 2) [14, 20, 21, 25, 26, 28, 30-32, 34]. The potential for misclassification bias was considered high in the remaining studies as they relied on self-report for medication exposure $[18,22,23,27,29,33,35,36]$ and/or did not provide references supporting the inclusion of medicines as potentially alcohol interactive [19, 23, 24, 29, 33, 35].

While all studies relied on self-reported alcohol consumption, thus introducing potential biases in recall and reporting, 11 studies were considered to have a lower risk of bias as they reported on both quantity and frequency of alcohol consumption within a specified recall period, ranging from 1 week to 12 months (Table 2) $[14,18-23,25,26,31,34]$. The risk of misclassification bias, specifically underestimating exposure to alcohol, was 


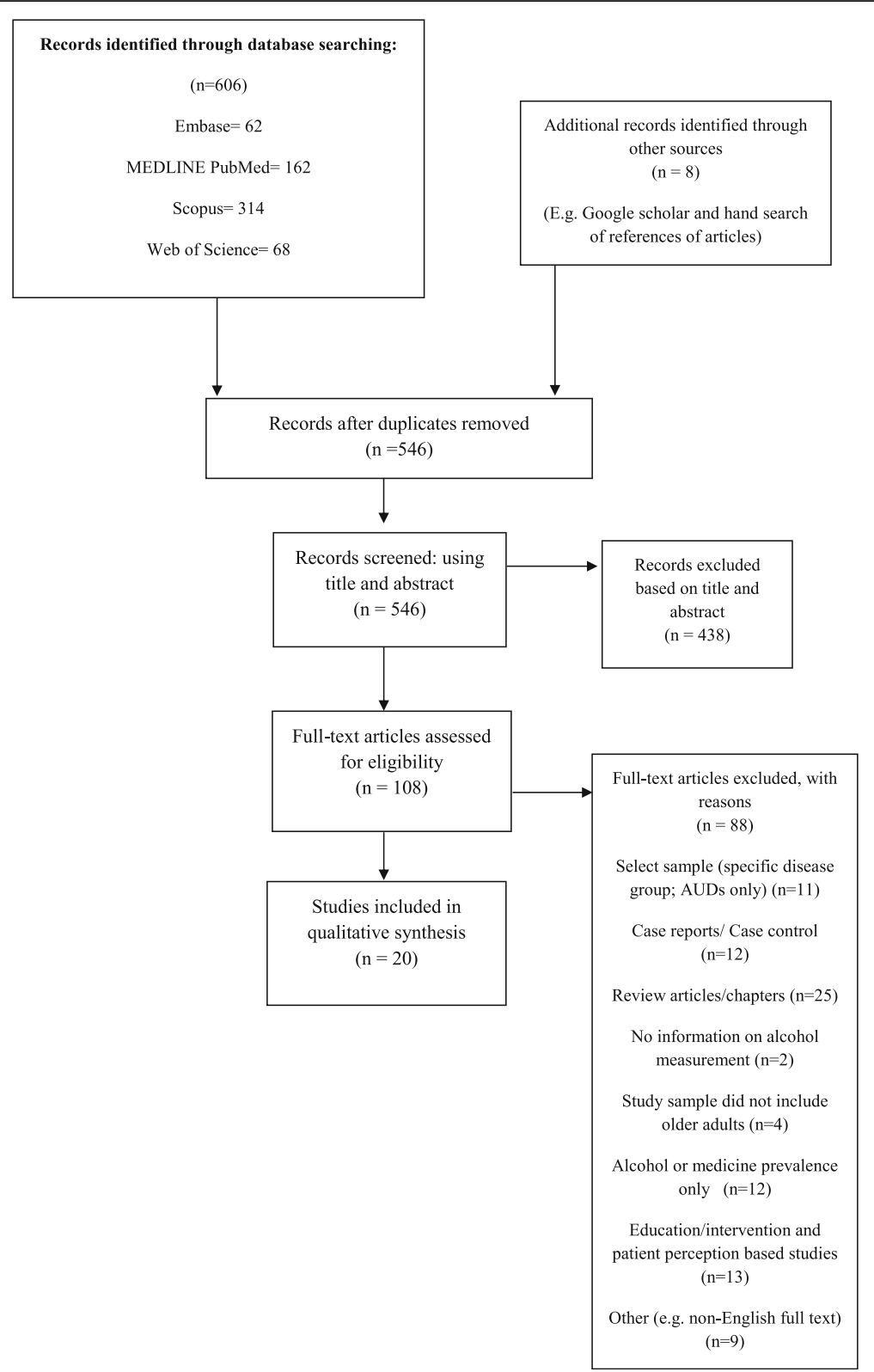

Fig. 1 Flow diagram of studies included in this review

considered to be higher in the remaining studies as they used quantity or frequency measures alone [24, 32, 35], did not specify the recall period $[24,27,30,35]$, restricted measurements to wine consumption $[29,36]$ and used a very narrow recall period "last working day or last weekend" [28]. Ascertainment of exposure to alcohol was unclear in one study [33].

In relation to outcome assessment, no study directly measured the concurrent use of alcohol and AI medications, rather all studies inferred concurrent use. Although possible for all studies, the risk of misclassifying the outcome of concurrent use was considered lowest in those studies identified as having a low risk of misclassifying exposure, who used the same recall period for both exposures or inferred concurrent use based on alcohol consumption within a specific recall period, ranging between 1 week and 12 months, and current or regular medication use (Table 2) $[14,20,21,25,26,31,34]$.

\section{Summary of findings}

As noted in Table 1, all estimates relate to studies from North America, Europe and Australia.

\section{Alcohol consumption}

The prevalence of alcohol consumption ranged between 57 and 63\% in studies reporting on nationally representative 


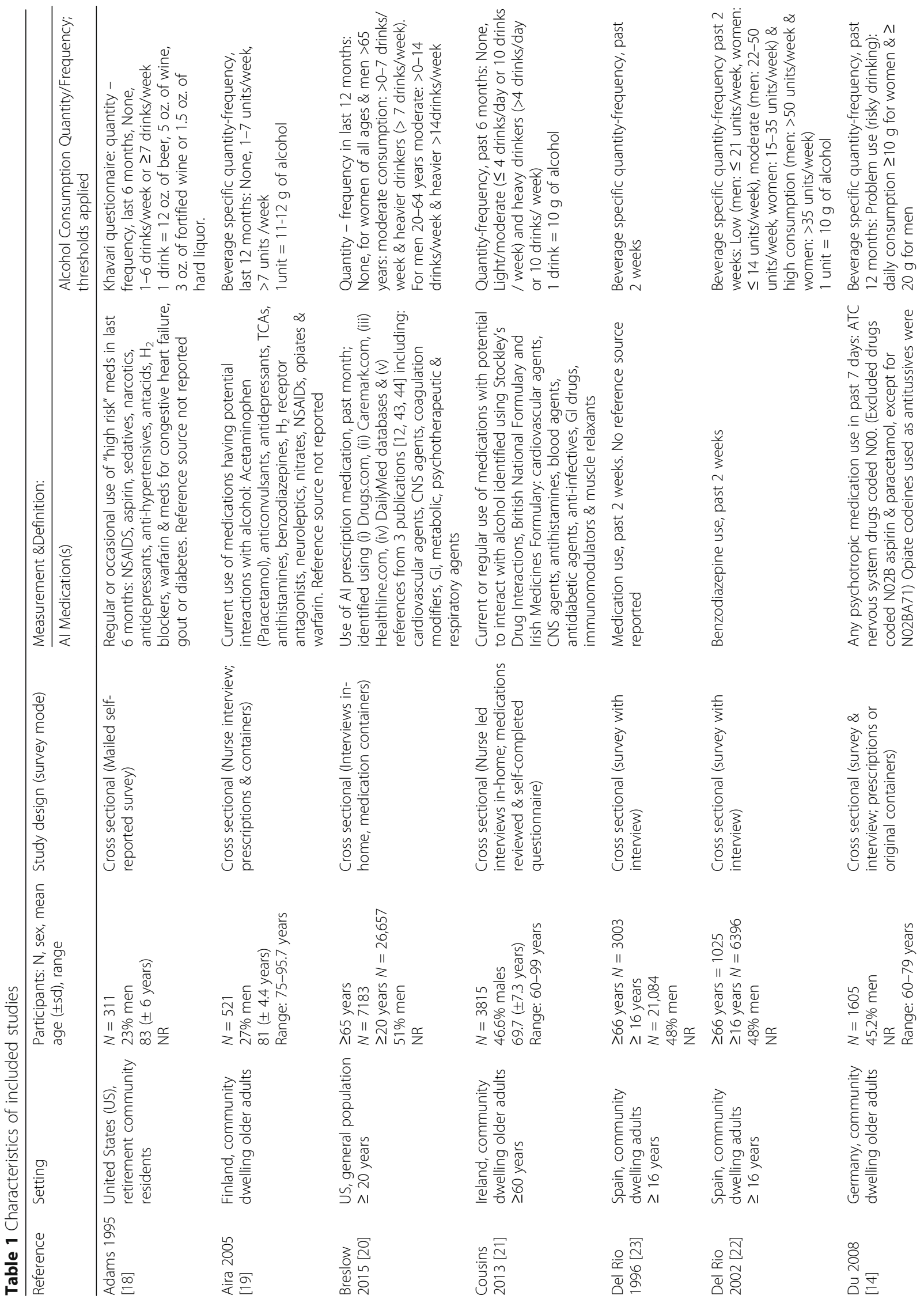




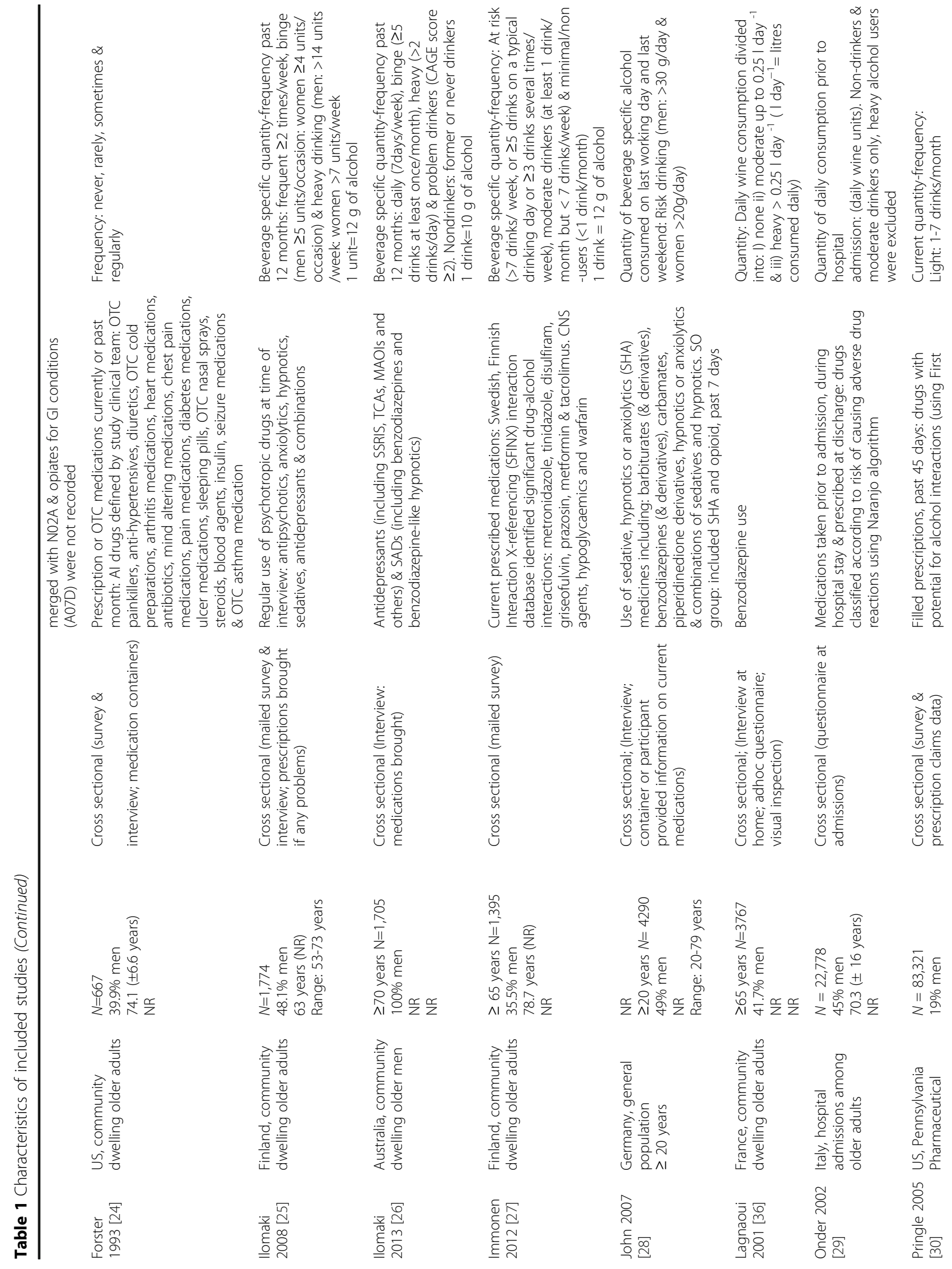




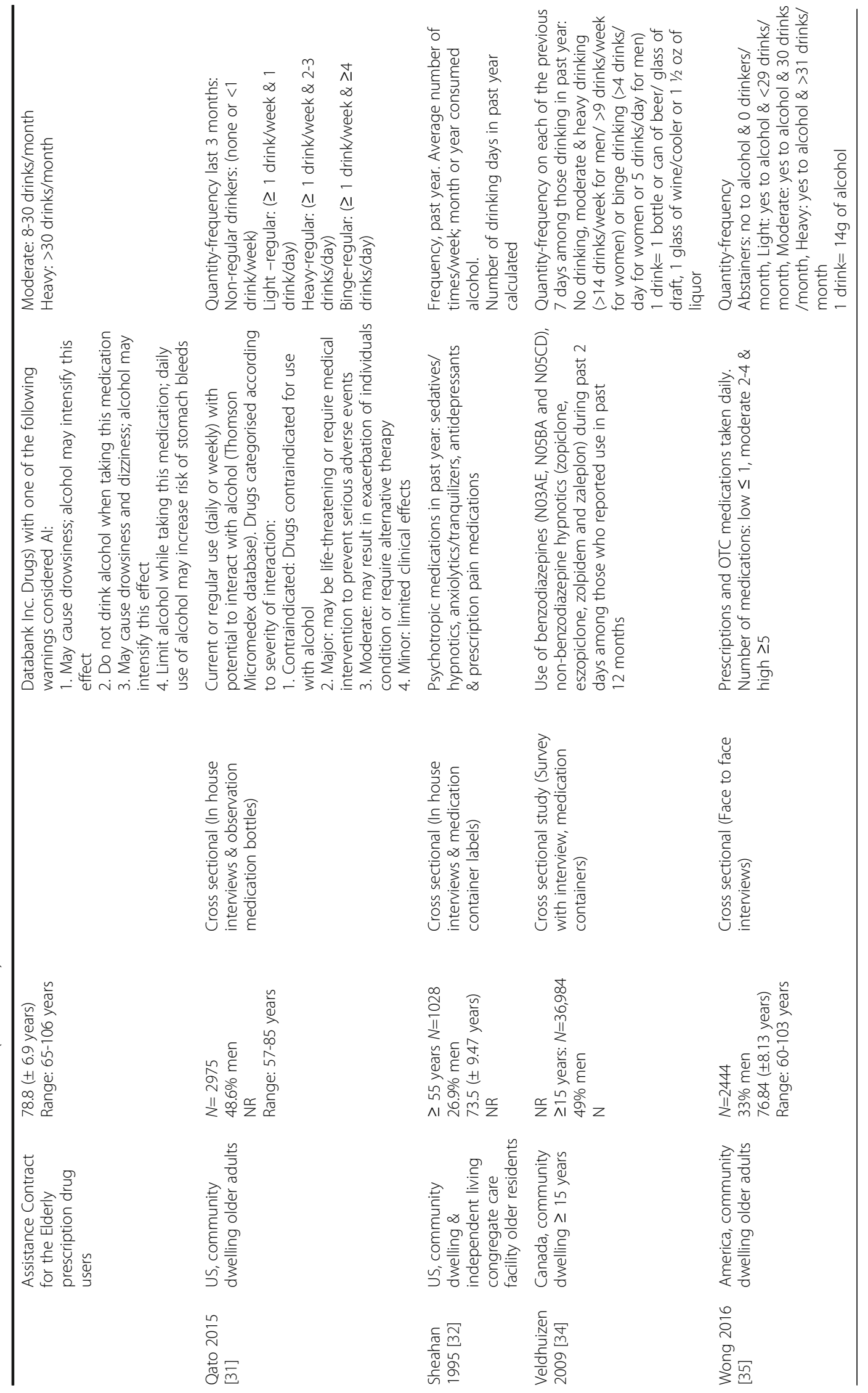


Table 2 Results of the critical appraisal of included studies

\begin{tabular}{|c|c|c|c|c|c|c|}
\hline Study & $\begin{array}{l}\text { Representativeness } \\
\text { of sample }\end{array}$ & $\begin{array}{l}\text { Ascertainment of } \\
\text { alcohol consumption }\end{array}$ & $\begin{array}{l}\text { Ascertainment of } \\
\text { Al medications } \\
\text { (classified) }\end{array}$ & $\begin{array}{l}\text { Ascertainment of } \\
\text { Al medications } \\
\text { (measured) }\end{array}$ & $\begin{array}{l}\text { Assessment of } \\
\text { the outcome } \\
\text { (concurrent use) }\end{array}$ & Study total: \\
\hline \multicolumn{7}{|c|}{ a) Cross Sectional Studies Outcome of Interest Concurrent use of alcohol and medications: } \\
\hline Adams et al. 1995 [18] & & ++ & + & & & +++ \\
\hline Aira et al. 2005 [19] & + & ++ & & ++ & & +++++ \\
\hline Breslow et al. 2015 [20] & ++ & ++ & ++ & ++ & ++ & ++++++++++ \\
\hline Cousins et al. 2014 [21] & ++ & ++ & ++ & ++ & ++ & ++++++++++ \\
\hline Del Rio et al. 1996 [23] & + & ++ & & & & +++ \\
\hline Del Rio et al. 2002 [22] & + & ++ & ++ & & & +++++ \\
\hline Du et al. 2008 [14] & + & ++ & ++ & ++ & ++ & +++++++++ \\
\hline Forster et al. 1995 [24] & ++ & + & & ++ & & +++++ \\
\hline Ilomaki et al. 2008 [25] & + & ++ & ++ & ++ & ++ & +++++++++ \\
\hline Ilomaki et al. 2013 [26] & + & ++ & ++ & ++ & ++ & +++++++++ \\
\hline Immonen et al. 2012 [27] & ++ & + & ++ & & & +++++ \\
\hline John et al. 2007 [28] & + & + & ++ & ++ & & ++++++ \\
\hline Lagnaoui et al. 2001 [36] & & & ++ & & & ++ \\
\hline Onder et al. 2002 [29] & & + & & & & + \\
\hline Pringle et al. 2005 [30] & & + & ++ & ++ & & +++++ \\
\hline Sheahan et al. 1995 [32] & & + & ++ & ++ & & +++++ \\
\hline Qato et al. 2015 [31] & + & ++ & ++ & ++ & ++ & +++++++++ \\
\hline Swift et al. 2007 [33] & + & & & & & + \\
\hline Veldhuizen et al. 2009 [34] & + & ++ & ++ & ++ & ++ & +++++++++ \\
\hline Wong et al. 2016 [35] & & & & & & \\
\hline
\end{tabular}

samples of community dwelling older adults (Table 3) $[21,24,27]$. Prevalence estimates of alcohol consumption were generally lower in studies sampling restricted age-groups, for example estimates ranged between 33.7 and $44 \%$ for studies restricted to older adults aged $>70$ years [26] and $>75$ years [19]. Similarly, prevalence estimates of alcohol consumption were generally lower in those studies considered to have a high risk of selection bias [18, 29, 30, 32, 35]. For example, 20\% of older adults registered on the Pennsylvania pharmaceutical assistance contract for the elderly reported alcohol consumption [30]. All studies reporting on gender differences identified a higher prevalence of alcohol consumption in men [19-21, 25, 29, 31, 36].

\section{Alcohol interactive (Al) medicines}

Exposure to alcohol interactive medications varied across studies; nationally representative studies of community dwelling older adults using objective measures of AI exposure estimate exposure at between 72 and $79 \%$ among the total study samples (Table 3) [20, 21]. Studies with objective measures of exposure to psychotropic medications in community dwelling older adults reported prevalence estimates of between 11.5-20\% among the total study samples $[14,25,26]$. In contrast to alcohol consumption, use of psychotropic medications was significantly higher in women than men $[14,22,25,28,32]$.

\section{Concurrent use of alcohol and Al medications among older adults}

Eleven studies reported on the concurrent use of alcohol and alcohol interactive medications among the total study samples (Table 3) [14, 18-20, 23, 24, 31-33, 35, 36]. Eight of the 11 studies reported on alcohol consumption and a wide range of medicines with potential to interact with alcohol, with the prevalence of concurrent use ranging between 18 and $39 \%$ among the total study samples [18-20, 23, 24, 31, 33, 35]. Only two of these studies were considered to have a low risk of misclassification bias for concurrent use [20, 31] . Breslow et al. estimated the prevalence of concurrent use among older adults at 35\% [20]. Concurrent use was highest for cardiovascular agents (28\%) followed by metabolic agents (17\%), CNS agents (10\%) and coagulation modifiers (10\%). Approximately $7.75 \%$ of all older adults were identified as concurrent users of alcohol and psychotropic medications [20]. Qato et al. reported a prevalence estimate of $21 \%$, with potential alcohol-medication interactions in older adults aged between 57 and 84 years being significantly more 


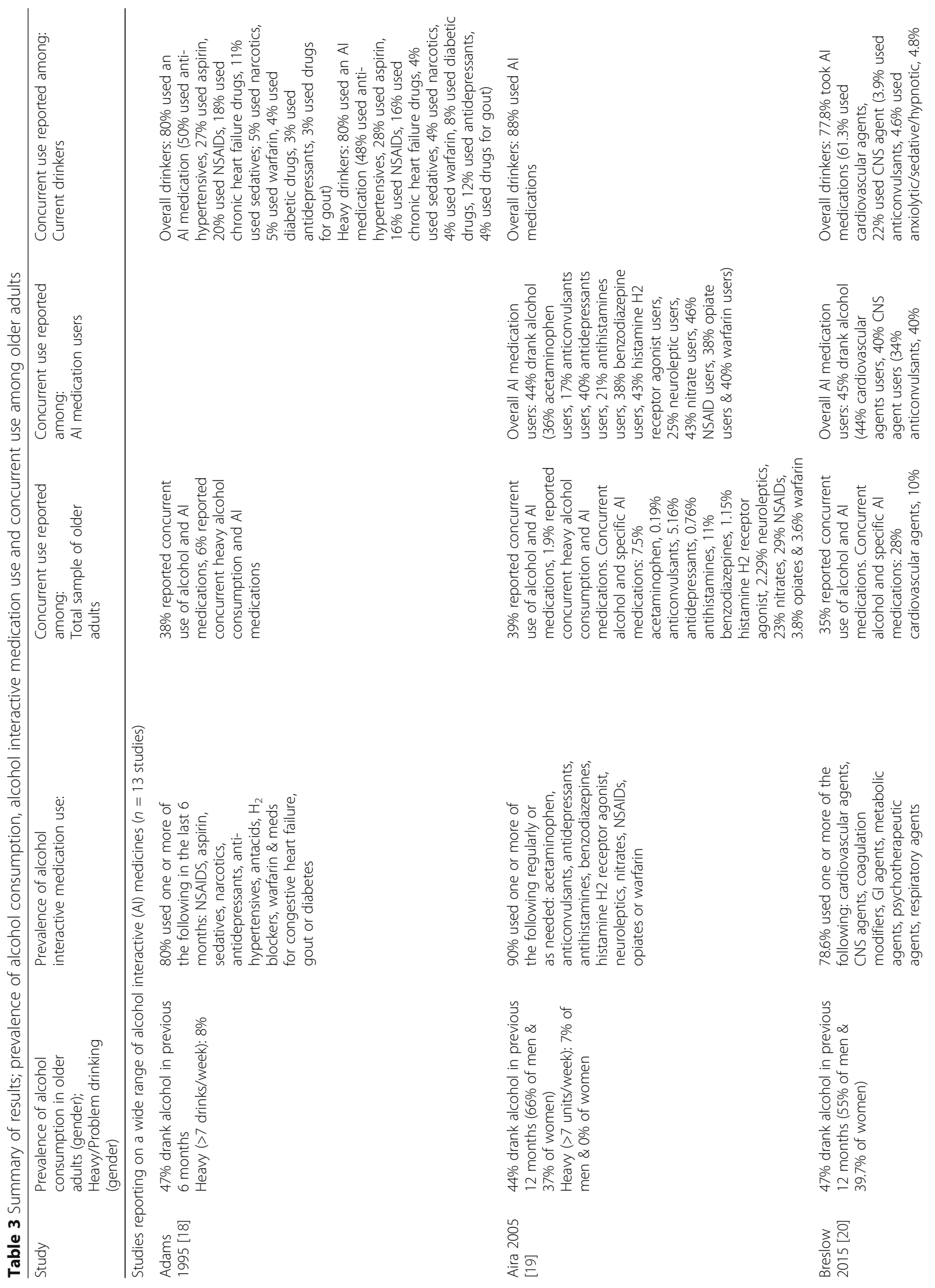



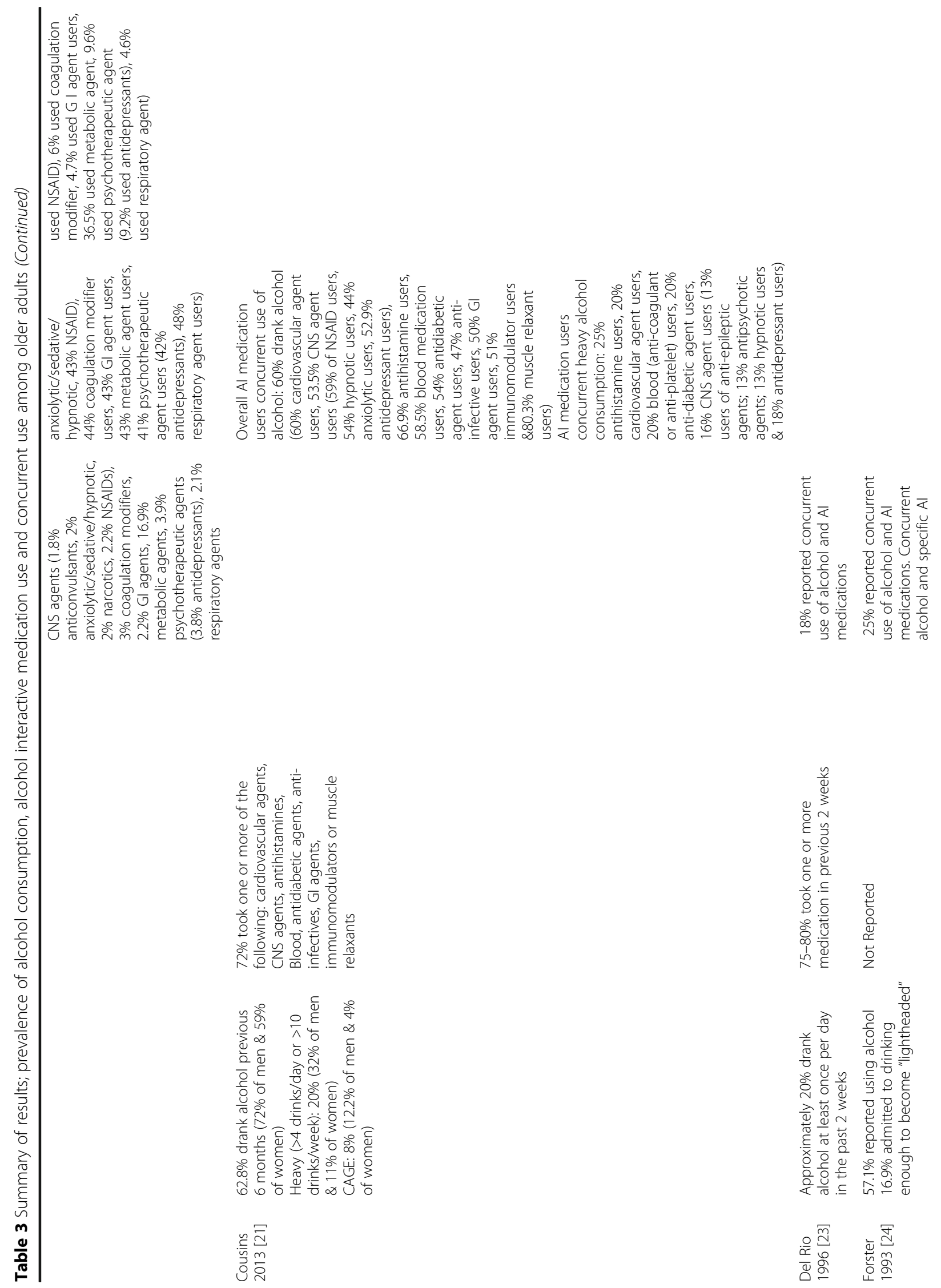

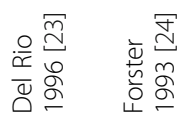




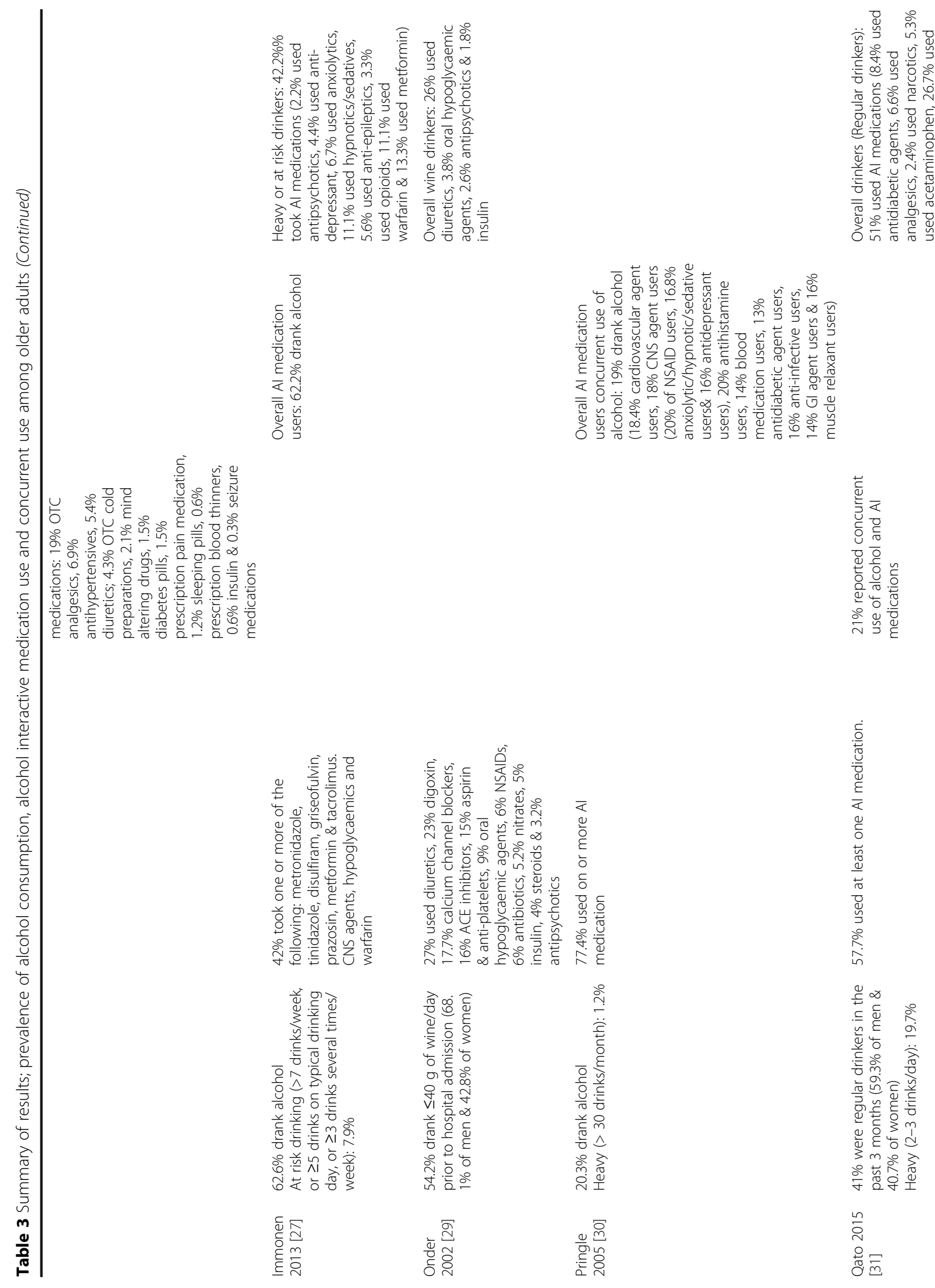



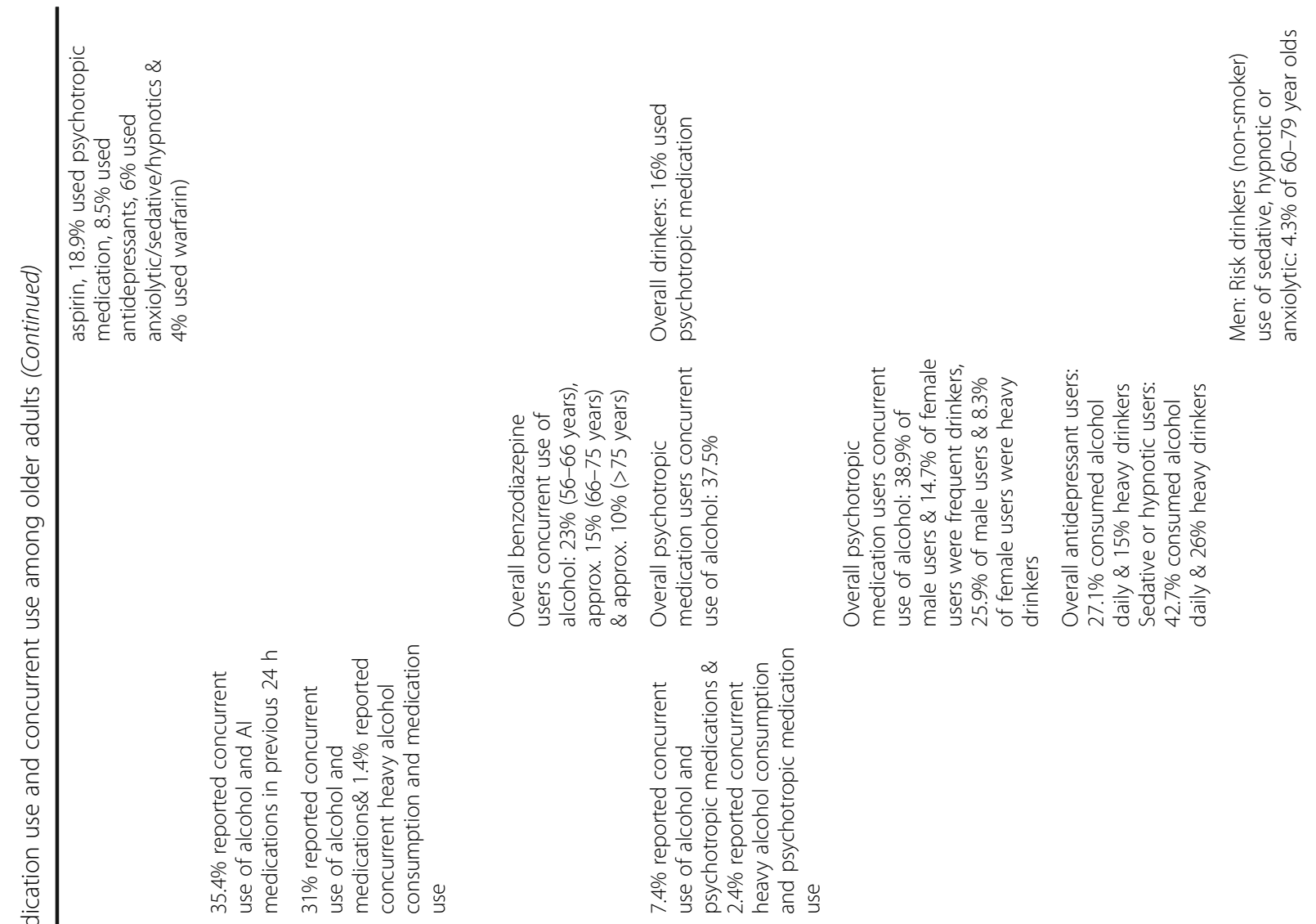

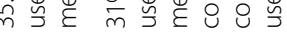
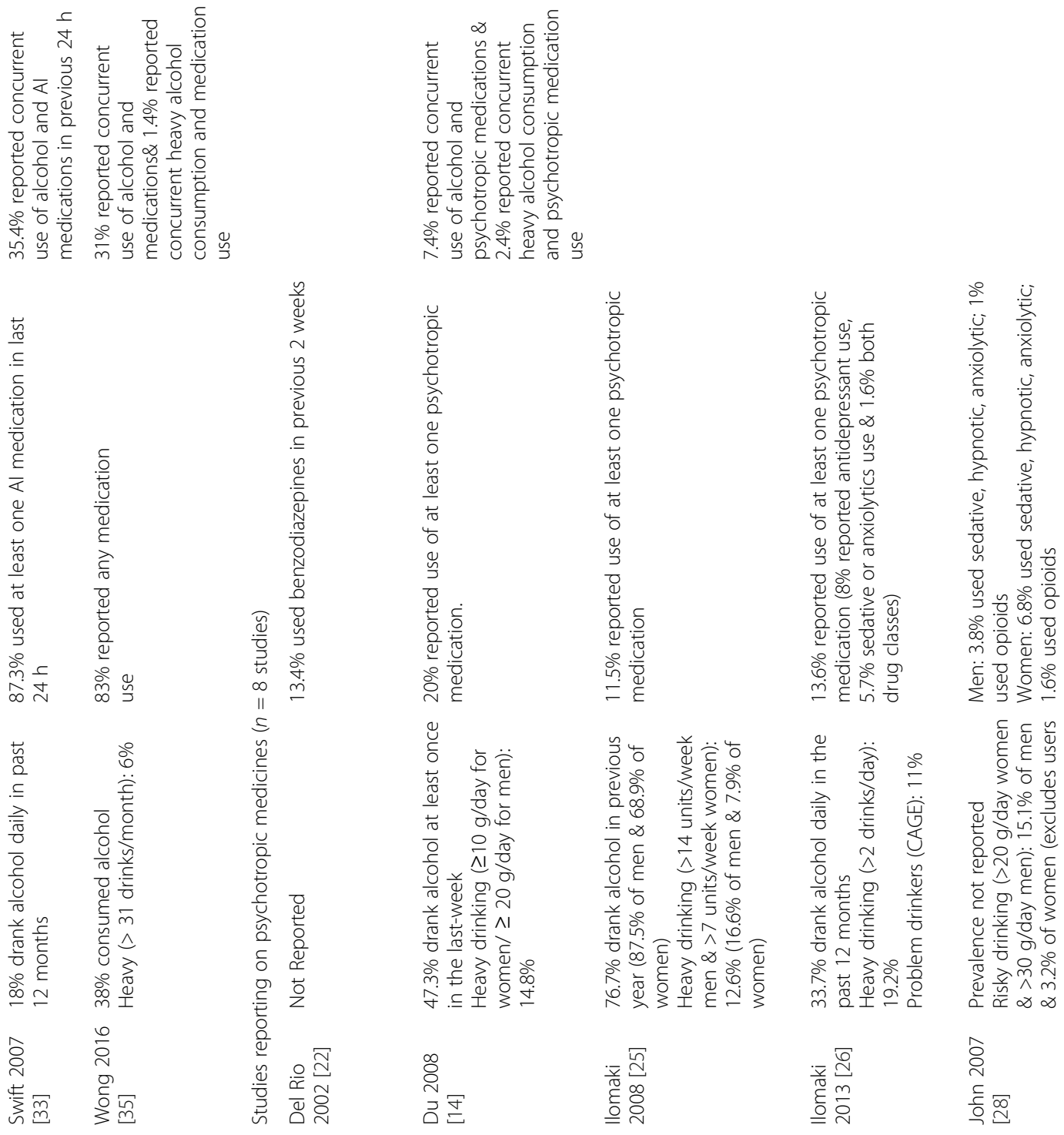

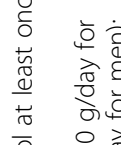

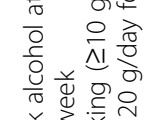

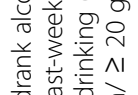

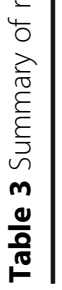

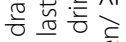

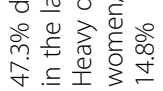
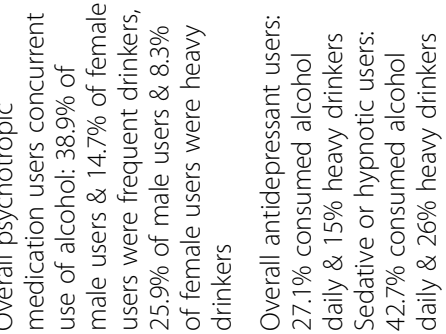

\section{要}




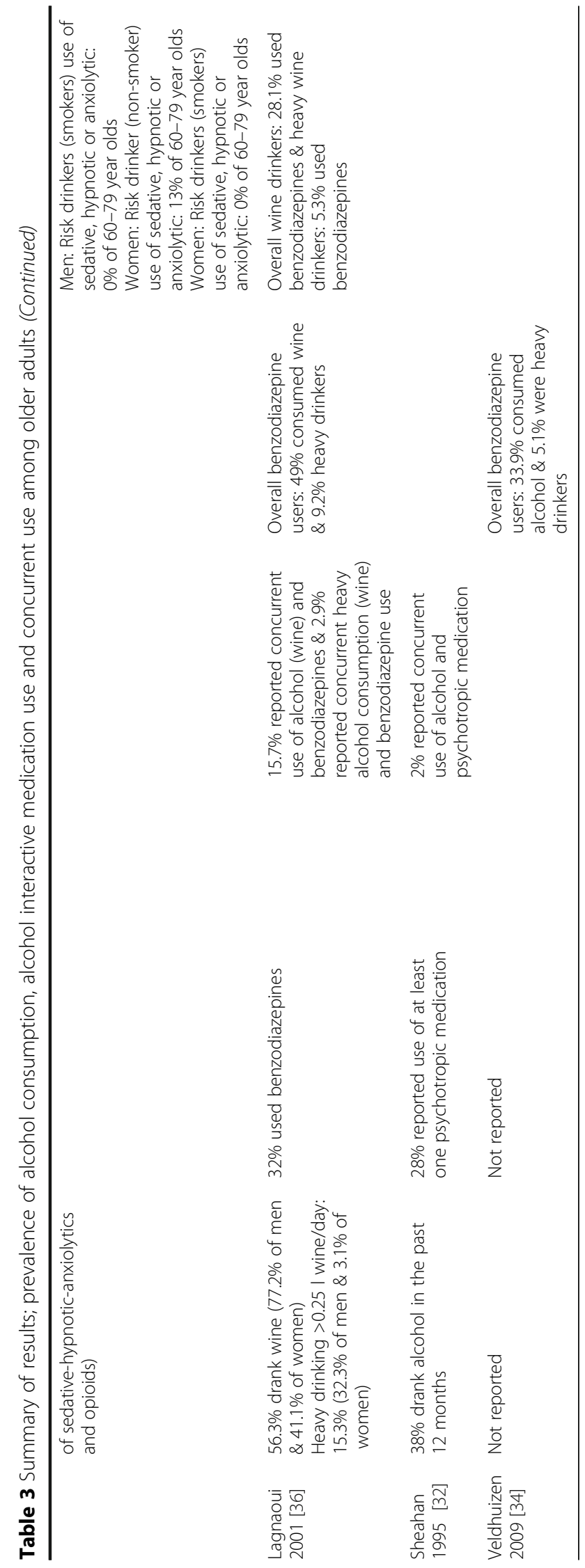


likely among men, white respondents, wealthier respondents, and those with higher education levels and greater comorbidities [31]. The prevalence of concurrent use was lower in studies which focused on psychotropic medications, ranging between 2 and 15.7\% $[14,32,36]$. Only one study was identified as having a low risk of misclassifying concurrent use in older adults [14], with an estimated prevalence of $7.4 \%$ among the total study sample and $2.4 \%$ for concurrent heavy alcohol consumption and psychotropic medications. Older age (70-79 years), residing in rural or small town areas, living alone, higher social status, polypharmacy and a poor social network were independently associated with concurrent use of alcohol and psychotropic medications [14]. While a higher proportion $(15.7 \%)$ of older adults in a French study [36] were identified as concurrent users of wine and benzodiazepines, the methods of ascertaining exposure to benzodiazepines was unclear, as was the assessment of concurrent use. Similarly, the lower estimate of $2 \%$ for concurrent use of alcohol and psychotropic medications in Sheahan et al. [32] is likely an artefact of their sample including patients in congregate care facilities.

Five studies assessed the prevalence of alcohol consumption among users of a broad range of alcohol interactive medications (Table 3) [19-21, 27, 30]. Breslow [20] and Cousins [21] were identified as having a low risk of misclassifying concurrent use, and reported prevalence estimates of 45 and $60 \%$ respectively. Cousins et al. [21] found that older adults using AI medications were significantly less likely to report alcohol consumption compared to those unexposed to AI medications. Younger age (60-64 years), men, urban dwelling, higher levels of education and a history of smoking were independently associated with concurrent use of alcohol and AI medications [21]. A further six studies focused on psychotropic medication users [14, 22, 25, 26, 34, 36], four of which were identified as having a low risk of misclassification bias [14, 25, 26, 34]. Du et al. estimated the prevalence of alcohol consumption among users of psychotropic medications at 37.5\% [14]. Similarly, Ilomaki et al. [25] reported that $38.9 \%$ of male psychotropic medication users, and $14.7 \%$ of their female users, consumed alcohol, with $26 \%$ of male and $8.3 \%$ of female users identified as heavy drinkers. Male psychotropic medication users consumed greater quantities of alcohol, and more often, than non-users. This pattern was not observed among women [25]. Between one-third and a half of all sedative or hypnotic users (34-54\%) have been shown to consume alcohol [20, 21, 26, 34] with between 5 and $13 \%$ drinking heavily [21, 34]. Consistent with these findings, Ilomaki et al. [15] found that $26 \%$ of men aged greater than 70 years who use sedatives or hypnotics drink heavily; heavy drinking and daily drinking was significantly higher among male sedative or hypnotic users compared to non-users [26]. Concurrent use of alcohol among older adults taking antidepressants ranged between 42 and 53\%, [20, 21] with $27 \%$ of male users aged greater than 70 years reporting concurrent use [26].

\section{Adverse outcomes associated with concurrent use of alcohol and alcohol interactive medications}

Only four studies reported on adverse outcomes. Three studies reported on falls, all three studies were crosssectional [27, 32, 35]. A study by Immonen et al. [27] of 2100 older adults in Finland found that falls and injuries when a person has consumed alcohol in the past 12 months were more common among at risk drinkers ( $>7$ drinks/week, or $\geq 5$ drinks drinking days or $\geq 3$ drinks several times/week) using AI medications (13.8\%) compared to AI medication-users who were not considered as at risk drinkers $(4.1 \%)(p<0.001)$ [27]. In contrast to these findings, Sheahan et al.'s [32] study of older adults in America, which included patients in congregate care facilities, found that although the number of psychotropic drugs was associated with an increased odds of falling, the concurrent use of alcohol and psychotropic drugs was not. Similar non-significant associations were reported in Wong et al.'s [35] convenience sample of older adults in the US. One study examined the association between moderate alcohol consumption $(\leq 40 \mathrm{~g}$ of wine per day) and adverse drug reactions among older adults at the point of admission to one of 81 acute care hospitals in Italy [29]. Among 22,778 participants, 3.9\% were identified as having one or more adverse drug reactions. Moderate alcohol consumption was associated with a $24 \%$ increase in the odds of having an adverse drug reaction [29].

\section{Discussion}

Overall, the results of this review suggest that between one-in-five and one-in-three older adults are potentially susceptible to alcohol-medication interactions, with more than half of AI medication users reporting alcohol consumption [20, 21, 31]. However, these estimates need to be interpreted with caution as studies differed in their classification of AI medications. In the absence of an explicit list of alcohol-interactive medications, multiple drug reference sources were used across studies [20, 21, 27, 30,31]. Use of different medication compendia led to a lack of consistency in the inclusion of AI medications across studies and may have led to the over or under inclusion of medications [31,37], resulting in an over-or-under estimate of concurrent use. There was however consensus with regards to CNS agents; all studies that reported on AI medicines classified CNS 
agents as alcohol interactive, specifically psychotropic medications [14, 18-22, 24-32, 34, 36]. Based on the quality assessment of studies, the most reliable estimates for the concurrent use of psychotropic medications and alcohol ranged between $7.4 \%$ [14] and 7.75\% [20].

Despite the high prevalence of concurrent use among older adults, no study examined longitudinal associations with adverse outcomes. Three cross-sectional studies reported on falls with mixed findings [27, 32, 35]; with one study reporting on an association between moderate alcohol consumption and adverse drug reactions among older adults at the point of admission to hospital [29]. An evidence based list of medications which have a significant risk of harm to older patients when combined with alcohol would be useful in a clinical setting, allowing for the identification of older adults whose alcohol consumption places them at increased risk and who would benefit from a preventative intervention. While recent studies have shown that clinicians rarely undertake screening and brief interventions to reduce alcohol consumption [38-40], flagging patients at the point of prescribing an alcohol-interactive medication may facilitate targeted screening and interventions to reduce harm. Brief alcohol interventions in primary care are effective in significantly reducing weekly alcohol consumption [41]. Additionally, educating older adults in relation to the risks associated with concurrent use of alcohol and medications has been shown to increase older adults' awareness of potential risks [42]. However, whether this intervention results in a behaviour change for those at risk is unclear [42].

This is the first systematic review to examine the prevalence of concurrent use of alcohol and alcohol interactive medications and associated adverse outcomes in older adults. An explicit and robust methodology was applied to identify, critically appraise and synthesise the study findings. However, the findings of the review need to be interpreted in the context of the study limitations. The risk of misclassification bias for both alcohol and AI medications was high across many studies, undermining internal validity. Furthermore, no study directly measured the concurrent use of alcohol and AI medications, rather all studies inferred concurrent use. Finally, heterogeneity across studies in relation to classifying medications as alcohol interactive and in the methods used to quantify alcohol consumption, prevent statistical pooling of data from existing studies. Variation in study setting and age restrictions, also make it difficult to compare prevalence of concurrent use across studies. Furthermore, due to the current gap in the literature, the available evidence for this review was restricted to three continents, Europe, North America and Australia.

\section{Conclusions}

While there appears to be a high propensity for alcoholmedication interactions in community dwelling older adults, there is a lack of consensus regarding what constitutes an alcohol interactive medication. An explicit list of alcohol interactive medications needs to be derived, and validated prospectively to quantify the magnitude of risk posed by the concurrent use of alcohol and alcohol interactive medications for adverse outcomes in older adults. This will allow for risk stratification of older adults at the point of prescribing, and prioritise alcohol screening and brief alcohol interventions in high-risk groups.

\section{Additional files}

Additional file 1: Database search: this document describes the retrieval process of studies for the systematic review. (DOCX $14 \mathrm{~kb}$ )

Additional file 2: Search strategy: This file describes the search strategies used in Embase, PubMed, Web of Science and Scopus in order to identify studies for this systematic review. (DOCX $15 \mathrm{~kb}$ )

Additional file 3: Newcastle-Ottawa Scale (NOS) adapted for crosssectional studies. (DOC $35 \mathrm{~kb}$ )

Additional file 4: Table S1. Most common alcohol interactive (Al) medicines across included studies. (DOCX 281 kb)

\section{Abbreviations}

ADRs: Adverse drug reactions; Al: Alcohol interactive; AUD: Alcohol use disorder; NSAIDs: Nonsteroidal anti-inflammatory drugs; OTC: Over the counter

\section{Acknowledgements}

Not applicable.

\section{Funding}

This study was funded by the Royal College of Surgeons in Ireland (RCSI), School of Pharmacy. The funder had no role in the design of the study and collection, analysis, and interpretation of data or in writing the manuscript.

Availability of data and materials

All data generated or analysed during this study are reported in the manuscript (and its Additional files 1, 2, 3 and 4).

\section{Authors' contributions}

$\mathrm{AH}$ defined the research question and the methodology including the search strategy, performed the literature search, and reviewed all included studies, extracted data, performed quality assessment and drafting of the manuscript. GC conceived the research study, defined the research question and methodology and reviewed full text articles, extracted data, and performed quality assessment. GC was also involved in writing of the paper and approved the final manuscript. TF defined the research question, and the methodology, reviewed the manuscript and approved this manuscript to be published. PG defined the research question, and the methodology, reviewed the manuscript and approved the manuscript to be published. All authors read and approved the final manuscript.

Ethics approval and consent to participate Not applicable.

Consent for publication

Not applicable.

Competing interests

The authors declare that they have no competing interests. 


\section{Publisher's Note}

Springer Nature remains neutral with regard to jurisdictional claims in published maps and institutional affiliations.

\section{Author details}

'School of Pharmacy, Royal College of Surgeons in Ireland (RCSI), Dublin 2, Ireland. ${ }^{2}$ HRB Centre for Primary Care Research, Department of General Practice, Royal College of Surgeons in Ireland (RCSI), Dublin 2, Ireland.

Received: 18 January 2017 Accepted: 4 July 2017

Published online: 17 July 2017

\section{References}

1. United Nations, Department of Economic and Social Affairs PD. World Population Prospects: The 2015 Revision, Key Findings and Advance Tables. New York: 2015.

2. Britton A, Ben-Shlomo Y, Benzeval M, Kuh D, Bell S. Life course trajectories of alcohol consumption in the United Kingdom using longitudinal data from nine cohort studies. BMC Med. 2015;13:47.

3. Meng Y, Holmes J, Hill-McManus D, Brennan A, Meier PS. Trend analysis and modelling of gender-specific age, period and birth cohort effects on alcohol abstention and consumption level for drinkers in great Britain using the general lifestyle survey 1984-2009. Addiction. 2014;109

4. Wadd S, Papadopoulos C. Drinking behaviour and alcohol-related harm amongst older adults: analysis of existing UK datasets. BMC Res Notes. 2014;7:741.

5. Moore AA, Whiteman EJ, Ward KT. Risks of combined alcohol/medication use in older adults. Am J Geriatr Pharmacother. 2007;5(1):64-74.

6. NHS Information Centre: Statistics for Alcohol: England 2010. 2011.

7. Office for National Statistics: 2010 Based Subnational Population Projections for England 2012.

8. Hovstadius B, Hovstadius K, Astrand B, Petersson G. Increasing polypharmacy an individual-based study of the Swedish population 2005-2008. BMC Clin Pharmacol. 2010;10:16.

9. Sumukadas D, McMurdo ME, Mangoni AA, Guthrie B. Temporal trends in anticholinergic medication prescription in older people: repeated cross-sectional analysis of population prescribing data. Age Ageing. 2014;43(4):515-21.

10. Richardson K, Moore P, Peklar J, Galvin R, Bennett K, RA. K. Polypharmacy in adults over 50 in Ireland: Opportunities for cost saving and improved healthcare. A report from The Irish Longitudinal Study on Ageing (TILDA). TILDA Trinity College Dublin 2012 December 2012. Report No.

11. Moriarty F, Hardy C, Bennett K, Smith SM, Fahey T. Trends and interaction of polypharmacy and potentially inappropriate prescribing in primary care over 15 years in Ireland: a repeated cross-sectional study. BMJ Open. 2015;5(9):e008656.

12. Weathermon R, Crabb DW. Alcohol and medication interactions. Alcohol Res Health. 1999;23(1):40-54.

13. Adams WL. Interactions between alcohol and other drugs. Int J Addict. 1995;30(13-14):1903-23.

14. Du Y, Scheidt-Nave C, Knopf H. Use of psychotropic drugs and alcoho among non-institutionalised elderly adults in Germany. Pharmacopsychiatry. 2008:41(6):242-51.

15. Ilomaki J, Paljarvi T, Korhonen MJ, Enlund H, Alderman CP, Kauhanen J, et al. Prevalence of concomitant use of alcohol and sedative-hypnotic drugs in middle and older aged persons: a systematic review. Ann Pharmacother. 2013;47(2):257-68.

16. Moher D, Liberati A, Tetzlaff J, Altman DG, Group P. Preferred reporting items for systematic reviews and meta-analyses: the PRISMA statement. Ann Intern Med. 2009;151(4):264-9. W64

17. Herzog R, Alvarez-Pasquin MJ, Diaz C, Del Barrio JL, Estrada JM, Gil A. Are healthcare workers' intentions to vaccinate related to their knowledge, beliefs and attitudes? A systematic review. BMC Public Health. 2013;13:154.

18. Adams WL. Potential for adverse drug-alcohol interactions among retirement community residents. J Am Geriatr Soc. 1995;43(9):1021-5.

19. Aira M, Hartikainen S, Sulkava R. Community prevalence of alcohol use and concomitant use of medication - a source of possible risk in the elderly aged 75 and older? Int J Geriatr Psychiatry. 2005;20(7):680-5.

20. Breslow RA, Dong C, White A. Prevalence of alcohol-interactive prescription medication use among current drinkers: United States, 1999 to 2010. Alcohol Clin Exp Res. 2015;39(2):371-9.

21. Cousins G, Galvin R, Flood M, Kennedy M-C, Motterlini N, Henman MC, et al. Potential for alcohol and drug interactions in older adults: evidence from the Irish longitudinal study on ageing. BMC Geriatr. 2014;14
22. Del Rio MC, Prada C, Alvarez FJ. Do Spanish patients drink alcohol while undergoing treatment with benzodiazepines? Alcohol. 2002;26(1):31-4.

23. Del Río MC, Prada C, Alvarez FJ. The use of medication and alcohol among the Spanish population. Br J Clin Pharmacol. 1996:41(3):253-5.

24. Forster LE, Pollow R, Stoller EP. Alcohol use and potential risk for alcoholrelated adverse drug reactions among community-based elderly. J Community Health. 1993;18(4):225-39.

25. Ilomaki J, Korhonen MJ, Enlund H, Hartzema AG, Kauhanen J. Risk drinking behavior among psychotropic drug users in an aging Finnish population: the FinDrink study. Alcohol. 2008;42(4):261-7.

26. Nomaeki J, Gnjidic D, Hilmer SN, Le Couteur DG, Naganathan V, Cumming $R G$, et al. Psychotropic drug use and alcohol drinking in communitydwelling older Australian men: the CHAMP study. Drug Alcohol Review. 2013;32(2):218-22

27. Immonen S, Pitkälä K. The prevalence of potential alcohol-drug interactions in older adults. Eur Geriatr Med. 2012;3:S134.

28. John U, Baumeister SE, Volzke H, Meyer C, Ulbricht S, Alte D. Sedative, hypnotic, anxiolytic and opioid medicament use and its co-occurrence with tobacco smoking and alcohol risk drinking in a community sample. BMC Public Health. 2007;7:337.

29. Onder G, Landi F, Della Vedova C, Atkinson H, Pedone C, Cesari M, et al. Moderate alcohol consumption and adverse drug reactions among older adults. Pharmacoepidemiol Drug Safety. 2002;11(5):385-92.

30. Pringle KE, Ahern FM, Heller DA, Gold CH, Brown TV. Potential for alcohol and prescription drug interactions in older people. J Am Geriatr Soc. 2005; 53(11):1930-6.

31. Qato DM, Manzoor BS, Lee TA. Drug-alcohol interactions in older US adults. J Am Geriatr Soc. 2015;63(11):2324-31.

32. Sheahan SL, Coons SJ, Robbins CA, Martin SS, Hendricks J, Latimer M. Psychoactive medication, alcohol use, and falls among older adults. J Behav Med. 1995;18(2):127-40.

33. Swift W, Stollznow N, Pirotta M. The use of alcohol and medicines among Australian adults. Aust N Z J Public Health. 2007:31(6):529-32.

34. Veldhuizen S, Wade TJ, Cairney J. Alcohol consumption among Canadians taking benzodiazepines and related drugs. Pharmacoepidemiol Drug Saf. 2009;18(3):203-10.

35. Wong $\mathrm{H}$, Heuberger $\mathrm{R}$, Logomarsino J, Hewlings S. Associations between alcohol use, polypharmacy and falls in older adults. Nurs Older People. 2016;28(1):30-6.

36. Lagnaoui R, Moore N, Dartigues JF, Fourrier A, Begaud B. Benzodiazepine use and wine consumption in the French elderly. Br J Clin Pharmacol. 2001; 52(4):455-6.

37. Vitry Al. Comparative assessment of four drug interaction compendia. $\mathrm{Br} \mathrm{J}$ Clin Pharmacol. 2007:63(6):709-14.

38. Brown J, West R, Angus C, Beard E, Brennan A, Drummond C, et al. Comparison of brief interventions in primary care on smoking and excessive alcohol consumption: a population survey in England. Br J Gen Pract. 2016; 66(642):e1-9.

39. Hamilton FL, Laverty AA, Gluvajic D, Huckvale K, Car J, Majeed A, et al. Effect of financial incentives on delivery of alcohol screening and brief intervention (ASBI) in primary care: longitudinal study. J Public Health. 2014;36(3):450-9.

40. Deehan A, Templeton L, Taylor C, Drummond C, Strang J. Low detection rates, negative attitudes and the failure to meet the "health of the nation" alcohol targets: findings from a national survey of GPs in England and Wales. Drug Alcohol Rev. 1998;17(3):249-58.

41. Kaner EF, Beyer F, Dickinson HO, Pienaar E, Campbell F, Schlesinger C, et al. Effectiveness of brief alcohol interventions in primary care populations. Cochrane Database Syst Rev. 2007;2:CD004148.

42. Benza AT, Calvert S, McQuown CB. Prevention BINGO: reducing medication and alcohol use risks for older adults. Aging Ment Health. 2010;14(8):1008-14.

43. National Consumers League (2013). Avoid Food Drug Interactions Food and Drug Administration Publication No. (FDA) CDER 10-1933.

44. Alcoholism NloAAa (2014). Harmful Interactions: mixing alcohol with medicines National Institutes of Health, US Department of Heath and Human Services 2014 